\title{
Are Monocyte/HDL, Lymphocyte/Monocyte and Neutrophil/Lymphocyte Ratios Prognostic or Follow-up Markers in Ischemic Cerebrovascular Patients?
}

 \\ 'University of Pamukkale School of Medicine, Department of Neurology, Denizli, Turkey \\ ${ }^{2}$ University of Pamukkale School of Medicine, Department of Biochemistry, Denizli, Turkey \\ ${ }^{3}$ University of Pamukkale School of Medicine, Department of Biostatistics, Denizli, Turkey \\ Address for Correspondence: Selma Tekin, E-mail: selmabilgintekin@gmail.com \\ Received: 11.112019. Accepted: 03.122019: Available Online Date: 27.012020 \\ (C) Copyright 2019 by Dokuz Eylül University, Institute of Health Sciences - Available online at www.jbachs.org \\ Cite this article as: Tekin S, Avcı E, Nar R, Değirmenci E, Demir S, Şenol H. Are Monocyte/HDL, Lymphocyte/Monocyte and Neutrophil/Lymphocyte Ratios Prognostic or Follow-up \\ Markers in Ischemic Cerebrovascular Patients? J Basic Clin Health Sci 2020; 1:38-43.
}

\begin{abstract}
Background: We investigated the association of monocyte to high-density lipoprotein cholesterol (HDL) ratio (MHR), neutrophil to lymphocyte ratio (NLR) and lymphocyte to monocyte ratio (LMR) with prognostic value and infarct types in patients with acute ischemic cerebrovascular disease (CVD).

Matherial and Methods: The study was carried out retrospectively in 223 patients, but after the exclusion criterion, 150 patients with acute ischemic CVD were included to the study. The complete blood count and lipid profile were examined at the admission of the patients. For initial neurologic status, National Institutes of Health Stroke Scale (NIHSS) score and for following-up, modified Rankin Scale (mRS) score were evaluated. After the etiological investigations and neuroimaging, infarct types were defined.

Results: We found that as the NIHSS score increased, LMR values decreased and NLR values increased statistically $(p=0.02, p=0.013$ ). Additionally, statistical significant differences were determined between MHR values and mRS scores ( $p=0.045)$. According to the results of regression analysis, it was observed that the increase of MHR was statistically significant on the MRS of people with cardioembolic infarction $(\mathrm{p}=0.004, \mathrm{StB}=0.383)$.

Conclusion: Our study provides, LMR and NLR values are related with the initial neurologic state, and they would be prognostic markers for the neurological deficit of acute cerebrovascular disease. MHR can be a follow-up marker for CVD and also a predictable marker for cardioembolic infarct type.
\end{abstract}

Key words: Acute ischemic cerebrovascular disease, monocyte to high-density lipoprotein cholesterol ratio, neutrophil to lymphocyte ratio, lymphocyte to monocyte ratio, prognosis.

\section{INTRODUCTION}

Ischemic cerebrovascular disease is a disorder which can be fatal or physically dysfunctional affecting both the person and the environment. According to the infarct types and related factors prognosis can be variable. Therefore, it is important to determine the prognostic factors in these patients.

Monocytes play an important role as pro-inflammatory and prooxidant cytokines in the immune system. In addition, monocytes are an important component of the atherosclerotic plaque structure. Therefore, high monocyte levels have been reported as an independent risk factor for coronary artery disease $(1,2)$. The protective effect of high-density lipoprotein (HDL) against lowdensity lipoprotein and monocyte oxidation is known (3). In recent years studies have shown that the ratio of monocyte/HDL (MHR) is a prognostic factor for coronary artery disease (4). In patients with intracerebral hemorrhage, the relationship between MHR value and mortality and disability has been examined and it has been shown that high MHR values play a prognostic role for mortality and disability after intracerebral hemorrhage (5). There is one study available in the literature that examines the MHR value and mortality in acute ischemic cerebrovascular disease. In this study, high MHR values have been shown to be independent predictors of mortality after acute CVD (6). However, the relationship between infarct subtypes and MHR has not been studied yet.

Moreover, lymphocytes, like monocytes, are important immunocytes in the development of atherosclerosis. It has been shown that low lymphocyte levels and high monocyte levels have important prognostic value in the development of acute myocardial infarction in cardiovascular diseases $(7,8)$. 
The lymphocyte-to-monocyte ratio (LMR) was a strong and independent predictor of long-term cardiac and cerebrovascular disease risk in patients with S-T elevated myocardial infarction (9). The relationship between LMR and acute cerebrovascular disease was examined and it was concluded that LMR may be a prognostic marker poor outcome (10).

In the present study, we aimed to investigate the prognostic value with simple and costless measurable indices like MHR, LMR and NLR in acute cerebrovascular disease. To the best of our knowledge this is the first study to evaluate the possible relationship between these indices and infarct subtypes.

\section{MATERIALS AND METHODS}

\section{Study Group}

We retrospectively investigated 223 patients with acute ischemic cerebrovascular disease who were hospitalized at Pamukkale University Neurology Department and intensive care unit in the first 24 hours of the CVD between the years 2014 and 2018. Ischemic stroke subtypes were classified according to the Trial of 10172 in Acute Stroke Treatment criterion (11). After the evaluation with exclusion criterion, 150 patients (81 males, 69 females were included to the study (Figure 1). The exclusion criterions were severe renal, liver, heart failure, malignancy, hematological disorder, systematic acute/chronic inflammatory/autoimmune or infectious diseases. Because of our study was a retrospective study, we did not take any written consent form from the patients. This study was approved by the Ethics Committee of Pamukkale University with the number of 60116787-020/32209.

\section{Data Collection}

$$
\begin{aligned}
& \text { Patients hospitalised with acute CVD } \\
& \text { in the first } 72 \text { hours between } 2014 \\
& \text { and } 2018 \text { at Pamukkale University }
\end{aligned}
$$$$
\text { Neurology Depertment }(n=223)
$$
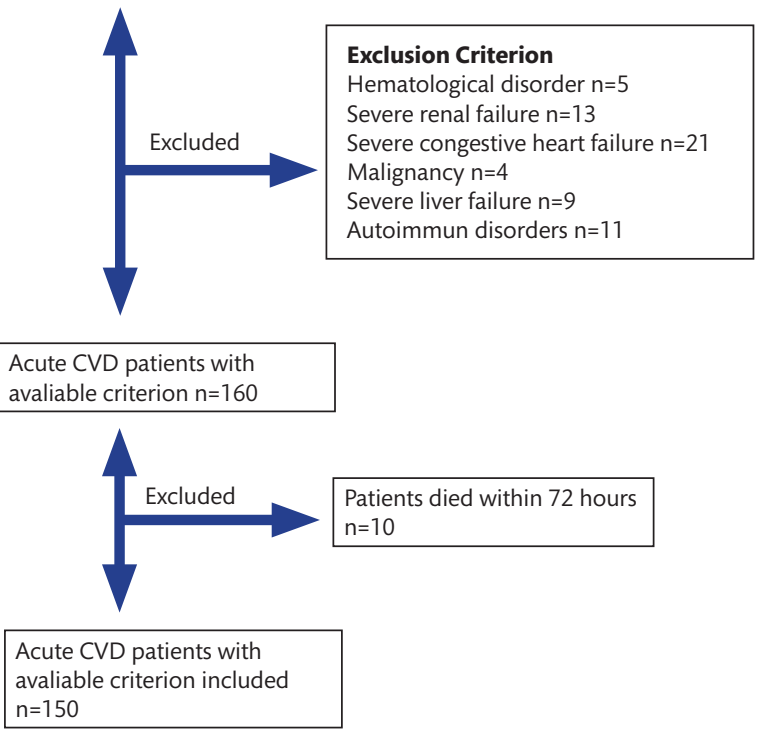

Figure 1. Features of included patients
Clinical data were collected for all patients, including demographic data (age, gender) and risk factors (hypertension, diabetes mellitus, hyperlipidemia, atrial fibrillation [AF], smoking, and heart disease). Cranial magnetic resonance (MR), or computed tomography (CT) imaging findings were used to show acute ischemic lesions.

Blood samples were taken within the 24 hours after the acute ischemic CVD. From each patient, approximately $8 \mathrm{ml}$ venous blood sample were drawn into serum separating and hemogram tubes.

Serum total cholesterol, LDL-cholesterol, HDL-cholesterol, creatinine, uric acid and ALT, AST levels were analyzed in Cobas e702 biochemistry auto-analyzer (Roche Diagnostics $\mathrm{GmbH}_{\text {, }}$ Mannheim, Germany). Complete blood counts were measured within $1 \mathrm{~h}$ of collection on a Siemens ADVIA 2120i hematology analyzer (Siemens Healthcare Diagnostics, Erlangen, Germany). MHR was calculated as the ratio of monocyte and HDL, LMR was calculated as the ratio of lymphocyte and monocyte lastly NLR was calculated as the ratio of neutrophil and lymphocyte, both obtained from the same automated blood sample at the same time.

\section{Stroke Severity and prognosis}

The patients' National Institutes of Health Stroke Scale scores (NIHSS) were measured on first admission. Stroke severity classified into 2 groups according to NIHSS values (NIHSS scores of 0-6 was defined as mild stroke and NIHSS scores $>6$ was defined as moderate to severe). After follow-up examinations stroke severity were measured with modified Rankin scale (mRS, scores range from 0 to 6 , when numbers increase, clinical outcome worsens.) before discharging.

\section{Statistical analysis}

Continuous variables were expressed as mean \pm standard deviation (SD), median (minimum-maximum values) and categorical variables as number and percent. Kolmogorov-Smirnov and Shapiro-Wilk tests were used for testing normality. If parametric test conditions were satisfied, Independent Samples t test was used for comparisons among groups. If parametric test conditions were not satisfied, Mann Whitney $U$ test was used for comparisons among groups. In addition, the relationships between continuous variables were analyzed by Spearman or Pearson correlation analysis and the differences between categorical variables were examined by Chi-square analysis. All statistical analyses analyzed by SPSS 24.0 (SPSS 24 for Windows, Armonk, NY: IBM Corp) and p value less than 0.05 was considered statistically significant.

\section{RESULTS}

Out of 223 patients, 150 patients were included in this study, after exclusion of 73 patients according to the exclusion criterion. The mean age was $67.69 \pm 13.44$ years. 81 of the patients $(54 \%)$ were male and 69 were female (46\%). Demographic and laboratory findings of the patients are shown in Table 1 and 2.

Moderate to severe strokes on admission were found in 34 patients (22.7\%), and the other 116 patients (77.3\%) were found to have mild stroke according to NIHSS scores. 
Table 1. Demographic-clinical findings and risk factors of patients

\section{NIHSS Score}

$\leq 6$

\begin{abstract}
Gender
\end{abstract}
Smoking

Diabetes Mellitus

Hypertension

Hyperlipidemia

Myocardial Infarction

Congestive Heart Failure

Atrial fibrillation

Stroke etiologic subtype

male/female
$-/+$
$-/+$
$-/+$

64 (\% 55.17)/52 (\% 44.83)

$87(\% 75) / 29(\% 25)$

$82(\%$ 70.69)/34 (\% 29.31)

40 (\% 34.48)/76 (\% 65.52)

90 (\% 77.59)/26 (\% 22.41)

$93(\%$ 80.17)/23 (\% 19.83)

$103(\% 88.79) / 13(\% 11.21)$

93 (\% 80.17)/23 (\% 19.83)

\begin{abstract}
31 (\% 26.72)
\end{abstract}
37 (\% 31.9)

21 (\% 18.1)

$15(\%$ 12.93)

12 (\% 10.34)
$>6$

\section{7 (\% 50)/17 (\% 50)} 25 (\% 73.53)/9 (\% 26.47)

21 (\% 61.76)/13 (\% 38.24)

14 (\% 41.18)/20 (\% 58.82)

29 (\% 85.29)/5 (\% 14.71)

$28(\% 82.35) / 6(\%$ 17.65)

$26(\%$ 76.47)/8 (\% 23.53)

21 (\% 61.76)/13 (\% 38.24)

$15(\% 44.12)$
$18(\% 52.94)$
$1(\% 2.94)$
$0(\% 0)$
$0(\% 0)$

P value

Total 0.595

$112(\% 74.67) / 38(\% 25.33) \quad 0.862$

$103(\%$ 68.67)/47 (\% 31.33) $\quad 0.324$

$54(\% 36) / 96(\%$ 64) $\quad 0.475$

$119(\%$ 79.33)/31 (\% 20.67) $\quad 0.329$

$121(\% 80.67) / 29(\%$ 19.33) $\quad 0.777$

$129(\% 86) / 21(\% 14) \quad 0.09$

$114(\% 76) / 36(\% 24) \quad 0.027^{*}$

$46(\% 30.67) \quad 0.0001^{*}$

55 (\% 36.67)

$22(\% 14.67)$

$15(\% 10)$

$12(\% 8)$

NIHHS: National Institues of Health Stroke Scale scores; TIA: Transient ischemic attack.

*Significant

Table 2. The quantative features of patients



NIHSS: National Institues of Health Stroke Scale scores; MRS: modified Rankin scale; LDL: low-density cholesterol; WBC: white blood cell; RBC: red blood cell; MCV: mean corpuscular volume; MCH: mean corpuscular volume; MCHC: mean corpuscular hemoglobin concentration; RDW: red blood cell distribution width.

*Significant 


\begin{tabular}{|c|c|c|c|c|c|c|}
\hline & Atherosclerotic (1) & Cardioembolic (2) & Lacunar (3) & Cyriptogenic (4) & TIA (5) & P value \\
\hline Age & $68 \pm 11.46$ & $71.4 \pm 12.32$ & $66.18 \pm 12.32$ & $53.93 \pm 17.44$ & $69.5 \pm 12.32$ & $0.005^{*}(2-4)$ \\
\hline NIHSS & $5.46 \pm 3.37$ & $5.78 \pm 3.9$ & $3.14 \pm 1.52$ & $2.67 \pm 1.76$ & $2.08 \pm 1.62$ & $0.0001^{*}(1-5,2-5,1-4,2-4,2-3)$ \\
\hline MRS & $2.04 \pm 1.71$ & $2.22 \pm 1.82$ & $1.55 \pm 1.47$ & $0.8 \pm 0.86$ & $0 \pm 0$ & $0.0001^{*}(1-5,2-5,3-5,2-4)$ \\
\hline Total Cholesterol (mmol/L) & $173.2 \pm 40.68$ & $168.44 \pm 42.31$ & $201.77 \pm 46.89$ & $170.93 \pm 36.98$ & $176.08 \pm 31.5$ & $0.033^{*}(2-3)$ \\
\hline LDL-cholesterol (mmol/L) & $104.24 \pm 32.91$ & $99.91 \pm 35.39$ & $115.09 \pm 47.31$ & $99.93 \pm 32$ & $104.58 \pm 26.8$ & 0.555 \\
\hline HDL-cholesterol (mmol/L) & $43.39 \pm 11.61$ & $44.53 \pm 15.26$ & $46.59 \pm 14.18$ & $39.07 \pm 7.28$ & $45.67 \pm 10.22$ & 0.562 \\
\hline VLDL-cholesterol (mmol/L) & $25.39 \pm 11.03$ & $23.28 \pm 11.1$ & $34.85 \pm 21.14$ & $31.93 \pm 12.76$ & $25.75 \pm 15.87$ & $0.036^{*}(2-4,2-3)$ \\
\hline Triglyceride (mmol/L) & $127.37 \pm 55.28$ & $120.15 \pm 50.29$ & $200.68 \pm 130.21$ & $159.93 \pm 63.44$ & $129.25 \pm 79.1$ & $0.013^{*}(2-3)$ \\
\hline Total WBC (109/L) & $11.4 \pm 18.0$ & $8.8 \pm 3.3$ & $8.5 \pm 2.6$ & $9.1 \pm 2.2$ & $8.2 \pm 2.2$ & 0.885 \\
\hline $\mathrm{RBC}\left(10^{9} / \mathrm{L}\right)$ & $4.78 \pm 0.68$ & $4.53 \pm 0.55$ & $5.03 \pm 0.71$ & $4.64 \pm 0.53$ & $4.64 \pm 0.58$ & $0.025^{*}(2-3)$ \\
\hline Hemoglobin (g/L) & $13.76 \pm 1.84$ & $13.23 \pm 1.56$ & $14.25 \pm 1.83$ & $13.51 \pm 1.55$ & $13.53 \pm 1.6$ & 0.183 \\
\hline
\end{tabular}

WBC: white blood cell; RBC: red blood cell; HGB: hemoglobine. ${ }^{*}$ Significant

Table 4. The significant correlation between NIHSS and serum lipid,

hematological markers

\section{NIHSS}

$\begin{array}{lc}\text { VLDL } & \mathrm{p}=0.042^{*} ; r=-0.167 \\ \text { TG } & \mathrm{p}=0.03^{*} ; r=-0.178 \\ \text { MCV } & \mathrm{p}=0.05^{*} ; r=-0.16 \\ \text { MCH } & \mathrm{p}=0.009^{*} ; r=-0.214 \\ \text { RDW } & \mathrm{p}=0.0001^{*} ; r=0.305\end{array}$

NIHSS: National Institues of Health Stroke Scale scores; LDL: low-density cholesterol; MCV: mean corpuscular volume; MCH: mean corpuscular volume; RDW: red blood cell distribution width. * Significant

The infarct sub-types were as follows: Atherosclerotic 46 (30.7\%); cardio embolic 55 (33.7\%); lacunar infarct 22 (14.7\%); cryptogenic $15(10 \%)$ and 12 patients (8\%) without infarct were included in the transient ischemic attack (TIA) group.

The initial NIHSS scores were significantly higher in cardio embolic infarct group than the lacunar and cryptogenic infarct group ( $p=0.0001$ ) (Table 3 ). Total cholesterol, VLDL, triglyceride, and RBC levels were found statistically higher in patients with lacunar infarcts than the other infarct subtypes $(p=0.03, p=0.03$, $\mathrm{p}=0.01, \mathrm{p}=0.02$ ) (Table 3).

VLDL, TG and lymphocyte values were significantly higher in mild strokes than the moderate to severe strokes. $(p=0.04, p=0.035$, $p=0.033$ ). But RDW values were significantly lower in mild strokes than the other group $(p=0.028)$ (Table 2$)$. There was positive correlation between NIHSS scores and RDW values ( $p=0.0001, r=0.305)$ and also there was a negative correlation between $\mathrm{VLDL}, \mathrm{TG}$ values with NIHSS scores ( $p=0.042 ; r=-0.167, p=0.03 ; r=-0.178$ ) (Table 4).

There was significant negative correlation between VLDL and TG values with mRS scores ( $p=0.01 ; r=-0.212, p=0.003 ; r=-0.238)$. In addition, we showed that poor outcome patients had lower VLDL and TG values. And there was significant positive correlation between RDW and mRS scores. The patients with good prognosis had lower RDW values ( $p=0.001 ; r=0.261$ ) (Table 5).

There was significant negative correlation between $\mathrm{MCV}, \mathrm{MCH}$ and NIHSS scores $\left(p=0.05^{*} ; r=-0.16, p=0.009^{*} ; r=-0.214\right)$. Statistical
Table 5. The significant correlation between MRS and biochemical parameters

\begin{tabular}{lc}
\hline $\mathrm{MCH}$ & MRS \\
RDW & $\mathrm{p}=0.021^{*} ; \mathrm{r}=-0.189$ \\
VLDL & $\mathrm{p}=0.001^{*} ; \mathrm{r}=0.261$ \\
TG & $\mathrm{p}=0.010^{*} ; \mathrm{r}=-0.212$ \\
& $\mathrm{p}=0.003^{*} ; \mathrm{r}=-0.238$
\end{tabular}

MRS: modified Rankin scale; MCH: mean corpuscular volume; RDW: red blood cell distribution width; VLDL: very low-density cholesterol; TG: trigyceride *Significant

Table 6. The comparisons of infarct types according to relation between MRS and MHR

\begin{tabular}{lcccccc}
\hline Dependent: MRS & $\begin{array}{c}\text { Std. } \\
\text { Beta }\end{array}$ & t & Sig. & $\begin{array}{c}\text { \% 95 C. } \\
\text { I. Lower } \\
\text { Bound }\end{array}$ & $\begin{array}{c}\text { \% 95 C. I. } \\
\text { Upper } \\
\text { Bound }\end{array}$ \\
\hline $\begin{array}{l}\text { Sub group } \\
\text { model 1 }\end{array}$ & Atherosclerotic & 0.090 & 0.620 & 0.540 & -0.050 & 0.100 \\
$\begin{array}{l}\text { Sub group } \\
\text { model 2 }\end{array}$ & Cardioembolic & 0.380 & 3.020 & $0.004^{*}$ & 0.040 & 0.190 \\
$\begin{array}{l}\text { Sub group } \\
\text { model 3 }\end{array}$ & Lacunar & -0.300 & -1.430 & 0.170 & -0.250 & 0.050 \\
$\begin{array}{l}\text { Sub group } \\
\text { model 4 } \\
\text { Independent: Monocyte/HDL ratio (MHR) }\end{array}$ & Cyriptogenic & 0.120 & 0.440 & 0.660 & -0.090 & 0.140 \\
\hline
\end{tabular}

significant difference was determined between monocytes and $m R S$ values $(p=0.017)$. The patients who had bad outcome, had higher monocyte levels. There was significant difference between lymphocyte/monocyte (LMR) ratio, neutrophil/lymphocyte ratio (NLR) and NIHSS scores $(p=0.02, p=0.013)$. As the NIHSS score increased, LMR and NLR values decreased statistically.

Statistical significant differences were determined between MHR values and mRS scores $(p=0.045)$. According to the results of regression analysis, it was observed that the increase of MHR was statistically significant on the MRS of people with cardioembolic infarction $(p=0.004, S t B=0.383)$. This effect was not significant in other infarct types (Table 6). 


\section{DISCUSSION}

Stroke is a disorder that can result with severe situations. If prognosis is predicted by the biomarkers, disability can be prevented earlier. There were many studies which evaluated stroke and the prognostic markers by the various hematological and biochemical parameters like the ratios; monocyte to highdensity lipoprotein (HDL) ratio (MHR), lymphocyte to monocyte ratio (LMR), neutrophil to lymphocyte ratio (NLR). Nevertheless, there is no study which shows the relationship between MHR, LMR, NLR and infarct types. In this study, we investigated the relationship between LMR, NLR and MHR values and prognosis in patients with ischemic stroke, and this is the first study to examine the relationship between these ratios and infarction subtypes.

In our study, cardio embolic infarct group (33.7\%) was more common than the atherosclerotic group in contrast to the common literature knowledge. Higher concentrations of plasma lipids can cause plaque formation, promoting artery stenosis and resulting ischemia. We found that statistical significant higher total cholesterol, VLDL, TG in patients with lacunar infarcts. These results were similar with the other studies in the literature $(11,12)$. VLDL and TG values were also higher in the patients with good prognosis. It may be related with the lacunar infarcts who have higher VLDL and TG and smaller infarct size, hence prognosis can be better than the patients who have larger infarcts. But some of the studies found that total cholesterol levels were not associated with lacunar infarcts $(13,14)$.

Our study demonstrates that patients with cardio embolic infarcts had lower RBC levels than the patients with lacunar infarcts. Hatamian et al. showed that RBC count could be used for to predict short term mortality in ischemic stroke (15). In our cardio embolic patients' population, initial neurologic examination was worse than the patient population of lacunar infarcts. According to this result we can conclude that lower RBC count may be a risk factor for poor prognosis in patients with cardio embolic infarcts than the patients with lacunar infarcts.

In our study, we found that RDW levels were correlated with NIHSS scores; higher RDW levels were associated with poor prognosis. Recent studies showed that elevated RDW values may cause stroke severity and independently predict future cardiovascular and allcause mortality $(16,17)$. Our data confirmed the results of these studies and RDW levels were significantly higher in moderate to severe stroke group.

We observed that $\mathrm{MCV}, \mathrm{MCH}$ values had negative correlation with NIHSS scores, hence lower MCV, MCH values were related with poor outcome. A. R Nayak et al. didn't find any significant alteration with these hematological parameters in acute ischemic stroke (18). Tzy-Haw et al. found that elevated MCV levels can be a predictor for the mortality of CVD and cardiovascular disease (19). But Hatamian et al. showed that lower MCV levels can be the predictors of short term mortality in ischemic stroke patients (15).

After ischemic brain damage, inflammatory response appears in damaged tissue. By the ischemia, oxygen-glucose deprivation causes releasing reactive oxygen species, complement factors and activated protease from the necrotic tissue (20). Experimental studies showed that after ischemia of the brain, pro-inflammatory cells like monocytes, $T$ cells, neutrophils are activated and over time they decrease while anti-inflammatory monocytes increase. By the way inhibiting the inflammatory response, infarct size begins to be smaller $(21,22)$. Bolayir et al. showed that higher monocyte counts' prognosis in acute CVD was worse than lower monocyte counts (6). In our study we also found that higher monocyte levels were related with poor prognosis too.

Leucocytes; predominantly neutrophils but also monocytes migrate within several hours to ischemic area. Infiltration of leucocytes occlude cerebral micro vessels and perfusion may be affected. Lymphocytes infiltrates later, macrophages are visible within 24 hours and decreasing of neutrophils are seen within 48 hours (20). The role of lymphocytes in the pathogenesis of ischemic stroke is unclear. Some studies claim that lymphocytes have a therapeutic effect on inflammation (23) but some of the other studies shows that lymphocytes have a pro-inflammatory effect and worsen the ischemic area. Kim et al. reported that lower lymphocyte levels are associated with poor prognosis (23).

However, to evaluate prognosis with more sensitive biomarkers, some ratios were calculated and investigated about inflammatory cells. For example, recent studies showed that neutrophil to lymphocyte ratio can be used as a predictor of prognosis for cardiovasculer disease, cancer, stroke and TIA (24-26). In our study, we also found that LMR and NLR values are lower in patients with worse neurological status. We thought that it was associated with stroke-induced immunosuppression which is related with the activation of the hypothalamus-pituitary axis and sympathetic nervous system (27). Stroke induced immunosuppression was defined by Liesz et al. with the effect of high mobility group box 1 (HMGB1) which is a nuclear protein synthesed from damaged brain tissue. This protein induces proliferation of immature monocytes from bone marrow through a receptor of advanced glycation end product (RAGE) signaling. And then immature monocytes secrete arginase-1 which is a marker of myeloid-derived suppressor cells and causes lymphocyte reduction by inducing lymphocyte apoptosis (28). Park et al. found that LMR was significantly lower in stroke patients with infections and LMR may be useful for assessing the stroke induced immunosuppression (29).

We showed that MHR values were statistically higher in the patient with higher mRS scores. And this is the first study which showed that the increase of MHR was statistically significant on the MRS of patients who had cardioembolic stroke, this effect was not significant in other infarct types. Higher HDL cholesterol levels are found to be associated with lower NIHSS score and good outcome (modified Rankin Scale score 0-1) in the literature (30-32). Lower HDL levels are risk factor for cardioembolic diseases. However, Hindy et al. found that elevated HDL levels lowered the risk of lacunar infarcts (33). According to the results of regression analysis, it was observed that the increase of MHR was statistically significant on the MRS of people with cardioembolic infarction $(p=0.004$, $\mathrm{StB}=0.383$ ). This effect was not significant in other infarct types. 
Our study has some limitations like relatively small patient population, because our study is a one-center-retrospective study. In addition, because of the study design the patients' follow-up period is too short, new prospectively with longer follow-up period would be helpful to predict long time disability in such patients.

In conclusion, this study provides, LMR and NLR values are related with the initial neurologic state, LMR and NLR are lower in patients with worse neurological status and they can be prognostic markers for the acute state of cerebrovascular disease. MHR can be a follow-up marker for CVD and also a predictable marker for cardioembolic infarct type.

\section{REFERENCES}

1. Nozawa N, Hibi K, Endo M, et al. Association Between Circulating Monocytes and Coronary Plaque Progression in Patients with Acute Myocardial Infarction. Circ J 2010;74:1384-1391. [CrossRef]

2. Afiune Neto A, Mansur Ade P, Avakian SD, Gomes EP, Ramires JA. Monocytosis is an Independent Risk Marker for Coronary Artery Disease. Arq Bras Cardiol 2006;86:240-244. [CrossRef]

3. Parthasarathy S, Barnett J, Fong LG. High-density lipoprotein inhibits the oxidative modification of low-density lipoprotein. Biochim Biophys Acta 1990;1044;275-283. [CrossRef]

4. Arısoy A, Altunkass, Karaman K, et al. Association of the Monocyte to HDL Cholesterol Ratio With Thrombus Burden in Patients With ST-Segment Elevation Myocardial Infarction. Clin Appl Thromb 2017;23:992-997. [CrossRef]

5. You S, Zhong C, Zheng D, et al. Monocyte to HDL cholesterol ratio is associated with discharge and 3-month outcome in patients with acute intracerebral hemorrhage. J Neurol Sci 2017;372:157-161. [CrossRef]

6. Bolayir A, Gokce SF, Cigdem B, et al. Monocyte/high-density lipoprotein ratio predicts the mortality in ischemic stroke patients. Neurol Neurochir Pol 2018;52:150-155. [CrossRef]

7. Núñez J, Núñez $E$, Bodí $V$, et al. Low lymphocyte count in acute phase of ST-segment elevation myocardial infarction predicts longterm recurrent myocardial infarction. Coron Artery Dis 2010;21:1-7. [CrossRef]

8. van der Laan AM, Hirsch A, Robbers LFHJ, et al. A proinflammatory monocyte response is associated with myocardial injury and impaired functional outcome in patients with ST-segment elevation myocardial infarction: Monocytes and myocardial infarction. Am Heart J 2012;163:57-65.e2. [CrossRef]

9. Wang Q, Ma J, Jiang Z, Wu F, Ping J, Ming L. Association of lymphocyteto-monocyte ratio with in-hospital and long-term major adverse cardiac and cerebrovascular events in patients with ST-elevated myocardial infarction. Med (United States) 2017;96:e7897. [CrossRef]

10. Ren H, Liu X, Wang L, Gao Y. Lymphocyte-to-Monocyte Ratio: A Novel Predictor of the Prognosis of Acute Ischemic Stroke. J Stroke Cerebrovasc Dis 2017;26:2595-2602. [CrossRef]

11. Lv $\mathrm{P}$, Jin $\mathrm{H}$, Liu $\mathrm{Y}$, et al. Comparison of risk factor between lacunar stroke and large artery atherosclerosis stroke: A cross-sectional study in China. PLoS One 2016;11:e0149605. [CrossRef]

12. Matz K, Tatschl C, Sebek K, Dachenhausen A, Brainin M. Dyslipidemia, elevated LDL cholesterol and reduced nocturnal blood pressure dipping denote lacunar strokes occurring during nighttime. Eur J Neurol 2004;11:742-748. [CrossRef]

13. Cui R, Iso $\mathrm{H}$, Yamagishi $\mathrm{K}$, et al. High serum total cholesterol levels is a risk factor of ischemic stroke for general Japanese population: The JPHC study. Atherosclerosis 2012;221:565-569. [CrossRef]

14. Zhang B, Zhang WZ, Li X, et al. Admission markers predict lacunar and non-lacunar stroke in young patients. Thromb Res 2011;128:1417. [CrossRef]

15. Hatamian H, Saberi A, Pourghasem M. The relationship between stroke mortality and red blood cell parameters. Iran J Neurol 2014;13:237-240. [CrossRef]
Compliance with Ethical Standards: Ethics Committee of Pamukkale University with the number of 60116787-020/32209

Peer-review: Externally peer-reviewed.

Author Contributions: Concept - ST, EA, RN; Design - ST, EA; Supervision - ST, ED, SD; Fundings - ST, EA; Materials - ST, EA, RN; Data Collection and/or Processing - ST, EA, HŞ; Analysis and/or Interpretation - ST, HŞ; Literature Search - ST, EA, RN; Writing Manuscript ST, EA; Critical Review - ST, ED, SD

Conflict of Interest: No conflict of interest was declared by the authors.

Financial Disclosure: The authors declared that this study has received no financial support.

16. Ani C, Ovbiagele B. Elevated red blood cell distribution width predicts mortality in persons with known stroke. J Neurol Sci 2009;277:103108. [CrossRef]

17. Lee $\mathrm{HB}, \mathrm{Kim} \mathrm{J}, \mathrm{Oh} \mathrm{SH}$, et al. Red blood cell distribution width is associated with severity of leukoaraiosis. PLoS One 2016;11:e0150308. [CrossRef]

18. Nayak AR, Kashyap RS, Kabra D, et al. Evaluation of routinely performed hematological and biochemical parameters for the prognosis of acute ischemic stroke patients. Neurol Sci 2011;32:855860. [CrossRef]

19. Niazi GA, Awada A, Rajeh S, Larbi E. Hematological values and their assessment as risk factor in Saudi patients with stroke. Acta Neurol Scand 1994;89:439-445. [CrossRef]

20. Kleinig TJ, Vink R. Suppression of inflammation in ischemic and hemorrhagic stroke: Therapeutic options. Curr Opin Neurol 2009;22:294-301. [CrossRef]

21. Jin R, Yang G, Li G. Inflammatory mechanisms in ischemic stroke: role of inflammatory cells. J Leukoc Biol 2010;87:779-789. [CrossRef]

22. Kim E, Yang J, Beltran CD, Cho S. Role of spleen-derived monocytes/ macrophages in acute ischemic brain injury. J Cereb Blood Flow Metab 2014;34:1411-1419. [CrossRef]

23. Li GZ, Zhong D, Yang LM, et al. Expression of interleukin-17 in ischemic brain tissue. Scand J Immunol 2005;62:481-486. [CrossRef]

24. Zhao L, Dai Q, Chen X, et al. Neutrophil-to-Lymphocyte Ratio Predicts Length of Stay and Acute Hospital Cost in Patients with Acute Ischemic Stroke. J Stroke Cerebrovasc Dis 2016;25:739-744. [CrossRef]

25. Tokgoz S, Kayrak M, Akpinar Z, Seyithanoğlu A, Güney F, Yürüten B. Neutrophil lymphocyte ratio as a predictor of stroke. J Stroke Cerebrovasc Dis 2013;22:1169-1174. [CrossRef]

26. Xue $\mathrm{P}$, Kanai M, Mori $\mathrm{Y}$, et al. Neutrophil-to-lymphocyte ratio for predicting palliative chemotherapy outcomes in advanced pancreatic cancer patients. Cancer Med 2014;3:406-415. [CrossRef]

27. Chamorro Á, Urra X, Planas AM. Infection after acute ischemic stroke: A manifestation of brain-induced immunodepression. Stroke 2007;38:1097-1103. [CrossRef]

28. Liesz A, Dalpke A, Mracsko E, et al. DAMP Signaling is a Key Pathway Inducing Immune Modulation after Brain Injury. J Neurosci 2015;35:583-598. [CrossRef]

29. Park MG, Kim MK, Chae SH, Kim HK, Han J, Park KP. Lymphocyteto-monocyte ratio on day 7 is associated with outcomes in acute ischemic stroke. Neurol Sci 2018;39:243-249. [CrossRef]

30. Putaala J, Strbian D, Mustanoja S, Haapaniemi E, Kaste M, Tatlisumak T. Functional outcome in young adult ischemic stroke: Impact of lipoproteins. Acta Neurol Scand 2013;127:61-69. [CrossRef]

31. Nardi K, Engelter S, Strbian D, et al. Lipid profiles and outcome in patients treated by intravenous thrombolysis for cerebral ischemia. Neurology 2012;79:1101-1108. [CrossRef]

32. Makihara N, Okada $\mathrm{Y}$, Koga $\mathrm{M}$, et al. Effect of serum lipid levels on stroke outcome after rt-PA therapy: SAMURAI rt-PA registry. Cerebrovasc Dis 2012;33:240-247. [CrossRef]

33. Hindy G, Engström G, Larsson SC, et al. Role of blood lipids in the development of ischemic stroke and its subtypes: A Mendelian randomization study. Stroke 2018:49:820-827. [CrossRef] 